\title{
Pathophysiologie, Diagnostik und Therapie von chronischem Husten: Neuronale Reflexe und Antitussiva
}

\section{Pathophysiology, Diagnostics and Therapy of Chronic Cough: Neuronal Reflexes and Antitussiva}

Autoren

Institute
Q. T. Dinh ${ }^{1,2}$, S. Heck ${ }^{2}$, D. D. Le ${ }^{2}$, R. Bals ${ }^{1}$, T. Welte ${ }^{3}$

Die Institutsangaben sind am Ende des Beitrags gelistet. eingereicht $\quad 8.2 .2013$ akzeptiert nach Revision 28.3.2013

\section{Bibliografie}

Dol http://dx.doi.org/ 10.1055/s-0033-1343151 Online-Publikation: 22.5.2013

Pneumologie 2013; 67: 327-334

(c) Georg Thieme Verlag KG

Stuttgart · New York

ISSN 0934-8387

\section{Korrespondenzadresse}

Prof. Dr. med. Quoc Thai Dinh

Klinik für Innere Medizin V, Pneumologie, Allergologie, Beatmungs- und Umweltmedizin und Abteilung für Experimentelle Pneumologie und Allergologie Universitätsklinikum und Medizinische Fakultät der Universität des Saarlandes Kirrberger Str, Gebäude 61.4 66421 Homburg/Saar thai.dinh@uks.eu www.uks.eu/expneu

\section{Zusammenfassung \\ $\nabla$}

Husten ist weltweit eines der häufigsten Symptome, welches zur Konsultation eines Arztes führt. Dabei stellt Husten einen wichtigen neuronalen Reflex dar, der als Schutzfunktion vor körperfremden Mikroorganismen, thermischen und chemischen Reizstoffen, die über die Atemwege eindringen können, dient. Zudem beugt er der Retention von Schleim in den Atemwegen vor. Der Husten-Reflex wird durch die Aktivierung verschiedener Husten-Rezeptoren initiiert. Diese Husten-Rezeptoren lassen sich hinsichtlich ihrer elektrophysiologischen Konfiguration in 3 Gruppen unterteilen, nämlich in die beiden $\mathrm{A \delta}-\mathrm{Fa}$ sern-Typen „schnell adaptierende (RAR) Mechanorezeptoren“ und „langsam adaptierende (SAR) Mechanorezeptoren“ und die C-Faser-Rezeptoren. Der Reiz wird durch die Husten-Rezeptoren über vagal-sensible Neuronen an das Husten-Zentrum im Gehirn weitergegeben. Der Husten selbst wird danach über Motoneuronen efferenter Nerven ausgebildet. Der Hustenreflex besteht somit aus 5 [1] funktionell aufeinander folgenden Teilen, nämlich den Husten-Rezeptoren [2], den primären afferenten Bahnen des N. vagus [3-5], N. trigeminus und N. glossopharyngeus [1], dem Hustenzentrum in der Medulla oblongata (N. tractus solitarius) [6-8], den afferenten Bahnen des $\mathrm{N}$. phrenicus, des Spinalnervs und des N. laryngeus recurrens und den Muskeln des Rachens sowie dem Zwerchfell und der abdominalen, intercostalen und laryngealen Muskulatur. Die Hustenrezeptoren befinden sich hauptsächlich in Larynx, Trachea und Hauptbronchien [2].

Der Vorgang des Hustens kann in 4 Phasen eingeteilt werden. Auf die erste Phase der schnellen Inspiration mit geöffneter Glottis folgen die Kompression mit geschlossener Glottis und ansteigendem trachealen Druck, die Akzeleration, bei der sich die Glottis öffnet, und schließlich die Exspiration/Expulsion mit geöffneter Glottis [9]. Gemäß

\section{Abstract \\ $\nabla$}

Cough is the number one symptom for patients to visit a physician worldwide. It is an important neuronal reflex which serves to protect the airways from inhaled exogenous microorganisms, thermal and chemical irritants. Moreover, it prevents the airways from mucus retention.

The cough reflex is initiated by activation of different cough receptors. These cough receptors can be divided into three groups according to their electrophysiological properties: into the two Adfiber types "rapid-adapting mechanoreceptor" (RAR) and "slow-adapting mechanoreceptor" (SAR), and the C-fiber receptor.

The stimulus is detected by cough receptors which conduct the signal to the cerebral cough centre via vagal-sensory neurons. The cough itself is mediated by efferent motoneurons. Hence the cough reflex consists of 5 functionally sequential parts [1]: the cough receptors [2], the primary afferent fibres of the N. vagus [3-5], N. trigeminus and $\mathrm{N}$. glossopharyngeus [1], the cough centre in the medulla oblongata (N. tractus solitarius) [6-8], the afferent fibres of the N. phrenicus, spinal nerve and N. laryngeus recurrens, as well as the diaphragm and the abdominal, intercostal and laryngeal muscles. The cough receptors are mainly located in the larynx, trachea and main bronchi [2]. The event of coughing can be divided into four subsequent parts: After the first phase of fast inspiration with an opened glottis, there is compression with a closed glottis and increasing tracheal pressure, acceleration and ultimately maximum expiration with an opened glottis [9]. According to its characteristics, cough can be split into two distinct types, "aspiration cough", which is loud and involuntary, and "urge-to-cough sensation", which describes an irritant, scratchy, and controlled cough of slowly increasing intensity [10]. Acute cough mostly develops because of infection of the respiratory system [11-13] and ends spon- 
seiner Charakteristik kann Husten in zwei distinkte Typen aufgeteilt werden, zum einen den „unfreiwilligen, lauten AspirationsHusten“, und zum anderen den kratzenden, reizenden und in seiner Intensität langsam ansteigenden kontrollierten Husten [10]. Akuter Husten entsteht häufig als Reaktion auf eine Infektion des respiratorischen Systems [11 - 13] und endet meist spontan nach 4 Wochen. Bis zu 8 Wochen hingegen kann Husten in chronischer Form durch einen infektiösen Befall mit Pathogenen wie Adenovirus, Bordetella pertussis und Mykoplasmen andauern [12 - 14]. Zur Unterteilung des Hustens nach seiner Ursache kann er auch nach Art und Weise differenziert werden. Zu unterscheiden sind trockener und Schleim-produzierender Husten.

Mit dieser Übersicht sollen die neuronalen Vorgänge und Mechanismen sowie Diagnostik und Therapie chronischen Hustens dargestellt werden. Dabei wird auch die Effizienz etablierter und potenzieller, noch nicht etablierter Antitussiva betrachtet.

$\begin{array}{ll}\text { Abkürzungsverzeichnis } \\ \text { ACE } & \text { Angiotensin Converting Enzyme } \\ \text { cAMP } & \text { cyclisches Adenosin-Mono-Phosphat } \\ \text { CB2 } & \text { Cannabinoid-Rezeptor 2 } \\ \text { CGRP } & \text { Calcitonin-Gene Related Peptide } \\ \text { COPD } & \text { Chronic Obstructive Pulmonary Disease } \\ \text { CVA } & \text { Cough-variant Asthma } \\ \text { DAG } & \text { Diaglycerol } \\ \text { GABA } & \text { Gamma-Amino-Butter-Acid } \\ \text { GÖR } & \text { Gastro-Ösophagealer Reflux } \\ \text { GqPC-B }{ }_{2}-\mathrm{R} & \text { G }_{\mathrm{q}} \text {-Protein-gekoppelter Bradykinin-2-Rezeptor } \\ \text { GsPC-PGE } 2 \text {-R } & \text { G }_{\mathrm{s}} \text {-Protein-gekoppelter Prostaglandin- } \mathrm{E}_{2} \text {-Rezeptor } \\ \text { IP } & \text { Inositol-1,4,5-triphosphat } \\ \text { NK-1 } & \text { Neurokinin-Rezeptor 1 } \\ \text { NK-2 } & \text { Neurokinin-Rezeptor 2 } \\ \text { NK-3 } & \text { Neurokinin-Rezeptor 3 } \\ \text { NOP1 } & \text { Nociceptin-Rezeptor 1 } \\ \text { PGE } 2 & \text { Prostaglandin-E } 2 \\ \text { PKA } & \text { Phosphokinase A } \\ \text { PLC } & \text { Phospholipase C } \\ \text { PND } & \text { Postnasales Drip-Syndrom } \\ \text { RAR } & \text { Rapidly Adapting Receptor } \\ \text { SAR } & \text { Slowly Adapting Receptor } \\ \text { TRPA1 } & \text { Transient Receptor Potential Ankyrin 1 } \\ \text { TRPV1 } & \text { Transient Receptor Potential Vanilloid 1 } \\ \text { TRPV4 } & \text { Transient Receptor Potential Vanilloid 4 } \\ \text { TRPM8 } & \text { Transient Receptor Potential Melastin 8 } \\ \text { VCD } & \text { Vocal Cord Dysfunction } \\ & \end{array}$

\section{Pathophysiologie des Hustens \\ $\nabla$}

Aס- und C-Fasern leiten die Stimuli der

Husten-Rezeptoren

Die Husten-Rezeptoren lassen sich hinsichtlich ihrer elektrophysiologischen Konfiguration in 3 Gruppen unterteilen, nämlich in

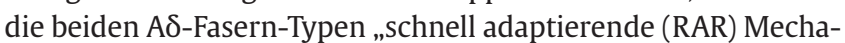
norezeptoren“ und „langsam adaptierende (SAR) Mechanorezeptoren" und die C-Faser-Rezeptoren. Die Aס-Fasern sind myelinisiert, weisen eine Leitgeschwindigkeit von 3-5 m/s [15] auf und spielen eine Schlüsselrolle in der Inhibition der Aspiration. Die zweite Gruppe Hustenreiz-vermittelnder Nervenfasern sind die C-Fasern, welche einen großen Teil des N. vagus ausmachen. Im taneously after 4 weeks. In contrast to this, bacterial infection with pathogens like Adenovirus, Bordetella pertussis and Mycoplasms can last up to 8 weeks [12 - 14]. In additional to the division of cough according to its cause, it can also be differentiated according to its manner: dry and mucus-producing cough.

With this review we want to give an overview of neuronal processes and mechanisms, as well as diagnostics of and therapy for chronic cough. Thereby the focus is also placed on the efficiency of already established and potential future antitussive agents.

Gegensatz zu den Ad-Fasern sind sie nicht myelinisiert und haben eine geringere Leitgeschwindigkeit $(0,3-2 \mathrm{~m} / \mathrm{s})$.

Die Perikarya der Mechanorezeptoren befindet sich im Ganglion nodosum, die der C-Fasern im Ganglion jugulare [16]. Die C-Fasern des Respirationssystems können nicht mechanisch stimuliert werden, eine Aktivierung kann aber durch Inhalation von Allergenen, Bradykinin, Capsaicin, chemischen Reizstoffen, hypertoner Kochsalzlösung, Ozon, proinflammatorischen Mediatoren, trockener Kaltluft und Zigarettenrauch erfolgen [17-19] ( $\bullet$ Abb. 1). Daher sind C-Fasern auch verantwortlich für die Vermittlung von kratzendem, reizendem, langsam ansteigendem Husten („urge-to-cough-sensation“) [13]. Durch die strikte funktionelle Unterscheidung von Aס- und C-Fasern in zwei distinkte Populationen der Husten-vermittelnden Nervenfasern dürfen beide Fasertypen nicht als jeweils autark funktionierendes System angesehen werden, da neue Ergebnisse Hinweise auf eine Interaktion von A 8 - und C-Fasern in deren gemeinsamen Verlauf über den N. vagus liefern. Die Stimuli, die von den verschiedenen

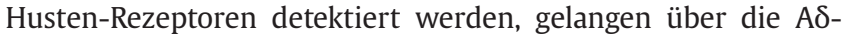
und C-Fasern durch den N. vagus zum Gehirn ( $\bullet$ Abb.2). Neben dem cerebralen Cortex beeinflussen sowohl die Insula als auch das anteriore Cingulum den Husten [20]. Der Einfluss dieser Hirnareale zeigt sich beim freiwilligen Husten bzw. bei der Unterdrückung des Hustens (cerebraler Cortex) [21-23] und darin, dass Husten in Anwesenheit von Anästhetika und im Schlaf nicht stattfindet $[24,25]$.

\section{Entzündungsmediatoren verursachen Husten}

Stimuli der Husten-Aktivierung sind Entzündungsmediatoren, wie Tachykinine, Bradykinin und Prostaglandine, die über unterschiedliche Mechanismen mit Entzündungsprozessen einhergehen. Diese können die Husten-Rezeptoren in den Atemwegen sensibilisieren und den Husten initiieren ( $\bullet$ Abb.1), auch verstärken. Bradykinin aktiviert den TRPV1-Rezeptor durch Bindung an den $\mathrm{G}_{\mathrm{q}}$-Protein-gekoppelten Bradykinin-2-Rezeptor $\left(\mathrm{G}_{\mathrm{q}} \mathrm{PC}-\mathrm{B2}\right.$ Rs), durch die Phospholipase C (PLC), welche die Diaglycerol (DAG)-Synthese stimuliert, und Inositol-1,4,5-triphosphat $\left(\mathrm{IP}_{3}\right)$. $\mathrm{IP}_{3}$ aktiviert seinen $\mathrm{IP}_{3}$-Rezeptor am Endoplasmatischen Retikulum. Dadurch werden $\mathrm{Ca}^{2+}$-Ionen ins Cytosol abgegeben. Prostaglandin (PGE2) aktiviert seine $\mathrm{G}_{\mathrm{s}}$-Protein-gekoppelten Prostaglandin- $\mathrm{E}_{2}$-Rezeptoren $\left(\mathrm{G}_{\mathrm{s}} \mathrm{PC}-\mathrm{PGE}_{2}-\mathrm{Rs}\right)$ und initiiert damit eine erhöhte Enzymaktivität der Adenylylcyclase und des zyklischen Adenosin-Monophosphats (cAMP), welches seinerseits die Proteinkinase A (PKA) aktiviert, was schließlich zur Phosphorylierung des TRPV1-Rezeptors führt. 


\section{TRP-Ionen-Kanäle und Husten}

Die Familie der Transient Rezeptor Potential (TRP) Ionenkanäle, zu der unter anderen TRPV1, TRPV4, TRPA1 sowie TRPM8 gehören, sind auf Nervenfasern und Zellmembranen der Perikarya exprimiert. Viele Studien konnten belegen, dass es durch Aktivierung der TRPV1- und TRPA1-Rezeptoren zu einer Modulation der sensiblen Neuronen und zu einer Auslösung des Hustenreflexes kommen kann [26]. Im Gegensatz dazu ist die Rolle der anderen TRP-Rezeptoren in den Atemwegen relativ unerforscht. TRPM8 wird durch Temperaturänderungen von harmloser zu schädlicher Kälte $\left(<15^{\circ} \mathrm{C}\right)$ sowie durch erfrischende Verbindungen, wie Menthol und Icilin, aktiviert [27]. In diesem Zusammenhang wird daher vermutet, dass TRPM8 als Ionenkanal für den Husten und die Bronchokonstriktion während der Einatmung von kalter Luft verantwortlich ist. Andere Studien zeigten wiederum antitussive Effekte von TRPM8 und lassen vermuten, dass Menthol den Hustenreflex unterdrückt. Obwohl die Wirksamkeit

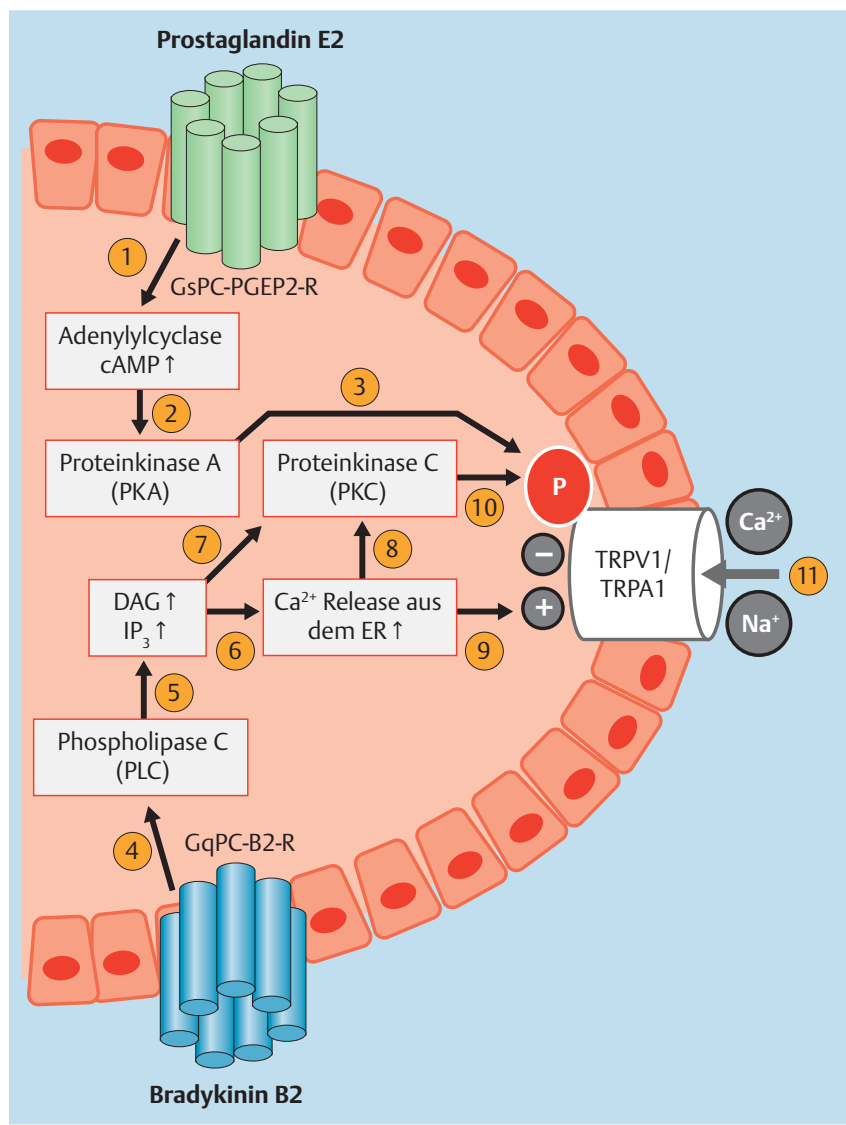

Abb. 1 Prostaglandine und Bradykinin verursachen Husten durch Aktivierung des TRPV 1 -Rezeptors bzw. TRPA 1 -Rezeptors. (1) Prostaglandin (PGE ${ }_{2}$ ) vermittelt über den $\mathrm{G}_{\mathrm{s}}$-Protein-gekoppelten Prostaglandin $\mathrm{E}_{2}$-Rezeptor $\left(\mathrm{G}_{\mathrm{s}} \mathrm{PC}-\mathrm{PG} \mathrm{E}_{2}-\mathrm{R}\right)$ eine erhöhte Enzymaktivität der Adenylylcyclase, was zur Erhöhung der CAMP-Produktion führt. (2) Das cyclische Adenosinmonophosphat aktiviert die Proteinkinase A (PKA), was die (3) Phosphorylierung des $\mathrm{TRPV}_{1}$-Rezeptors zur Folge hat [96]. (4) Bradykinin aktiviert die Phospholipase C (PLC) über den $G_{q}$-gekoppelten Bradykinin-2-Rezeptor ( $\left.G_{q} P C-B 2-R\right)$. (5) Die PLC stimuliert die Produktion von Inositol-1,4,5-triphosphat $\left(\mathrm{IP}_{3}\right)$ und Diaglycerol (DAG). (6) $I P_{3}$ aktiviert seinen $\mathrm{IP}_{3}$-Rezeptor am Endoplasmatischen Retikulum und initiiert somit die Freisetzung von $\mathrm{Ca}_{2+}$-lonen ins Cytoplasma und (9) die Modulation der TRPV ${ }_{1}-T_{R P A}$-Aktivität. (7) Erhöhte DAG-Konzentration und (8) $\mathrm{IP}_{3}$-Rezeptor-vermittelte $\mathrm{Ca}^{2+}$-Freisetzung aktivieren die Proteinkinase C (PKC), welche (10) TRPV $1 / T_{1}$ TPA $_{1}$ phosphoryliert. (11) Die Phosphorylierungen in (3) und (10) ändern die Konfiguration des $\mathrm{TRPV}_{1} / \mathrm{TRPA}_{1}$ und damit auch seine Permeabilität für $\mathrm{Ca}^{2+}$ - und $\mathrm{Na}^{+}$-lonen. von Menthol nicht erwiesen ist, besteht ein weit verbreiteter Gebrauch als Hustentherapeutikum [28]. Aussagekräftige epidemiologische Daten zum Bezug von Klima- und Temperaturänderungen und chronischem Husten sind zurzeit nicht verfügbar, sodass zukünftige Studien von großem Interesse wären.

\section{Klinische Einteilung des Hustens \\ $\nabla$}

\section{Akuter Husten}

Nach der Dauer wird Husten in akut, gelegentlich noch in subakut (Dauer 3-8 Wochen) und chronisch eingeteilt. Akuter Husten hält kürzer als 8 Wochen lang an. Er kann einziges Symptom bei lebensbedrohlichen Erkrankungen, wie bei Linksherzdekompensation, Lungenarterienembolie und Pneumothorax, sowie bei akut infektiösen und chronisch infektiösen Erkrankungen, wie Tuberkulose, die eine zeitnahe weitere Abklärung erfordert, sein. Mehr als 50\% der Patienten mit Lungenarterienembolie und Pneumothorax klagen über trockenen akuten Husten. Bakterielle und virale Infektionen in den oberen und unteren Atemwegen können den Husten auslösen [29-31]. Dabei liegen Hustenrezeptoren durch eine Verletzung des respiratorischen Epithels ungeschützt vor, was dazu führt, dass diese vermehrt gereizt und damit unspezifisch aktiviert werden. Zudem kann Husten auch bakteriell induziert werden, etwa durch Infektion mit B. pertussis oder mit C. diphtheriae [32] ( $\bullet$ Tab. 1$)$.

\section{Chronischer Husten}

Von chronischem Husten spricht man bei einer Dauer ab 8 Wochen. Chronischer Hustenreiz ist häufig Folge allergischer [33] oder nicht-allergischer Erkrankungen der oberen Atemwege. Allergie-induzierter Husten geht mit einem Symptomkomplex, bestehend aus Juckreiz in Augen, Nase und Rachen sowie Niesattacken, einher [1,34-37], chronischer Husten wird meist durch Laryngitis, Pharyngitis und Rhinitis ausgelöst [38-40] $(\bullet$ Tab.2). Die Inzidenz von Husten bei Erkrankungen der oberen Atemwege liegt, je nach gewählter Studie, zwischen 6-87\%. Chronischer Husten wird von Patienten häufig in Zusammenhang mit postnasalem Drip-Syndrom [41 - 43], intermittierender Nasenblockade und Gesichts- und Kopfschmerzen genannt.

Neben Dyspnoe und Obstruktion der Atemwege zählt Husten zu den Hauptsymptomen von Asthma bronchiale [44]. Außerdem kann chronischer Husten ein Anzeichen von COPD („Chronisch

Tab. 1 Respiratorische und extrapulmonale Erkrankungen mit akutem Husten.

Akut (<8 Wochen), subakut 3 - 8 Wochen
Erkrankungen der Atemwege:
- Sinusitis
- Rhino-Laryngo-Tracheobronchitis: meist virale Infektion oder
allergisch
- Asthma
- Aspiration: oft Kinder 1 - 3 Jahre
- inhalative Intoxikation: Unfälle, Brände
- postinfektiöser Husten
- Pneumonie
- Pleuritis
- Lungenembolie
- Pneumothorax
Extrapulmonale Ursachen:
- akute Linksherzinsuffizienz
- Bradykardie mit AV Block II, III




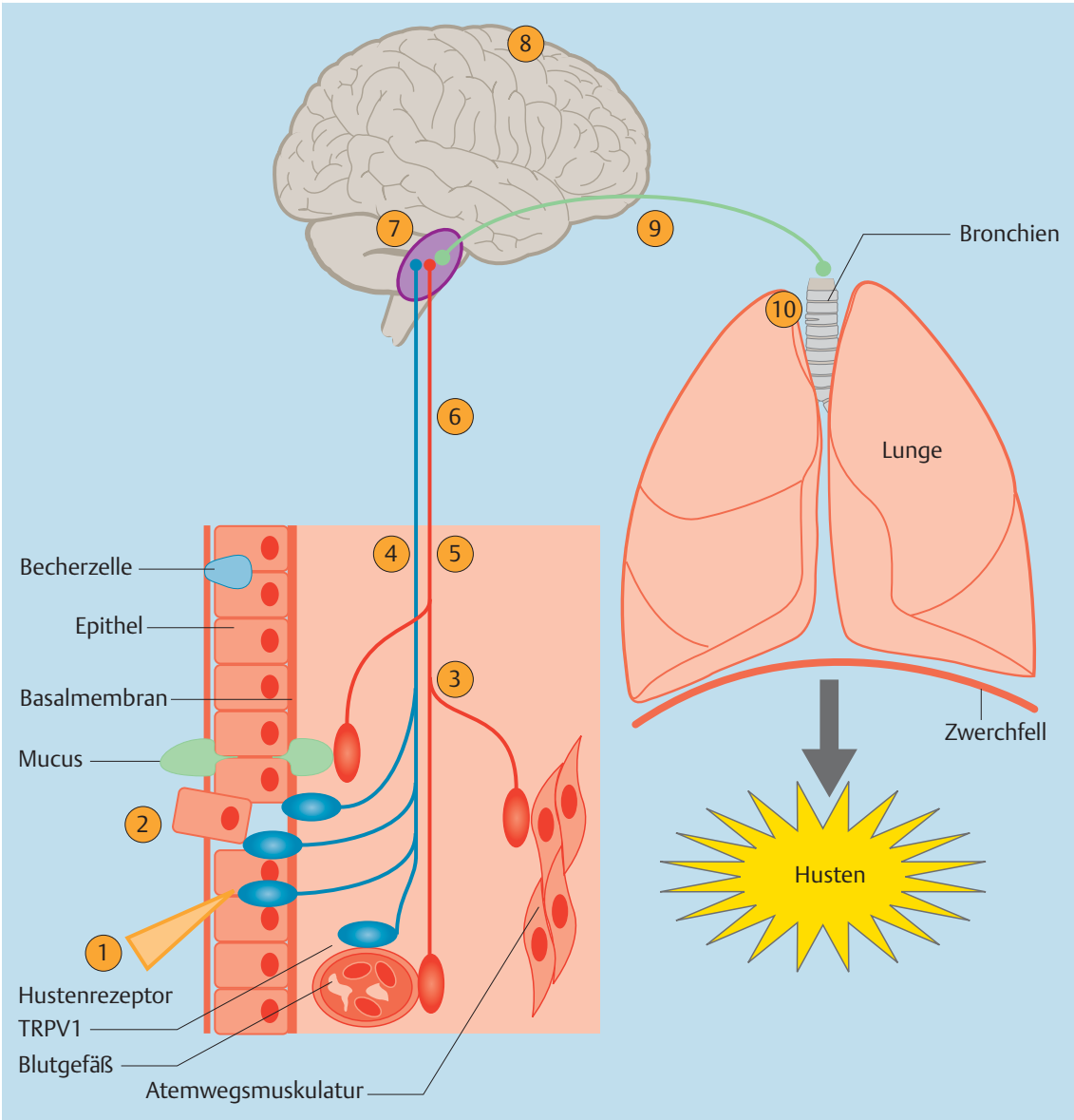

Abb.2 Schematische Illustration des Hustenreflexes in zeitlicher Reihenfolge. (1) Der Hustenreflex kann durch verschiedene Noxen, wie Entzündungen (Asthma, COPD, chronische und akute Infektionen), mechanische Reize durch Fremdkörper, durch Erkrankungen (Sarkoidose, Cystische Fibrose, Erkrankungen des Lungenparenchyms, Bronchiektasie), durch chemische Reize (Magensäure), Inhalation von Tabakrauch [97], Lungenkarzinome und Medikamente (ACE-Hemmer, Anticholinergika u. a.), ausgelöst werden. Auch epitheliale Schädigungen (2) resultieren in der Modulation der Hustenrezeptoraktivität durch Entzündungsmediatoren, wie (3) Histamin, Prostaglandine und TNF- $\alpha$. Die beiden Rezeptortypen (4) RAR (rapidly-adapting mechanoreceptor) und (5) SAR (slowly-adapting mechanoreceptor) sowie TRPV ${ }_{1}$ detektieren Reize und leiten die Information weiter. (6) Über den N. vagus gelangt der Reiz in das in der Medulla oblongata gelegene Hustenzentrum (7). Der cerebrale Cortex kann die motorische Aktivierung des Hustens beeinflussen (8). (9) Über Spinalafferenzen, den $\mathrm{N}$. phrenicus und den $\mathrm{N}$. laryngeus recurrens innerviert das Nervensystem (10) das Diaphragma und die Bauch-, Interkostal- und Laryngealmuskulatur und löst dadurch den Husten aus.

Obere und untere Atemwegserkrankungen

\begin{tabular}{|lll|}
\hline häufig & gelegentlich & selten \\
\hline Asthma bronchiale & Lungenkarzinom & exogene allergische Alveolitis \\
\hline bronchiale Hyperreaktivität & eosinophile Bronchitis & Bronchioektasien \\
\hline chronische Bronchitis & Pertussis & interstitielle Lungenkrankheit \\
\hline chronische Infektion & & Mukoviszidose \\
\hline COPD & pulmonale Radiotherapie \\
\hline PND (Postnasales Drip-Syndrom) & Sarkoidose \\
\hline obstruktive Schlafapnoe & tracheobronchialer Kollaps \\
\hline extrapulmonale Erkrankungen & Tuberkulose \\
\hline GÖR & VCD (Vocal Cord Dysfunction) \\
\hline psychogener Husten & \\
\hline chronisch idiopathischer Husten & \\
\hline kardiale Erkrankungen & \\
\hline (Herzinsuffizienz) & \\
\hline
\end{tabular}

Tab. 2 Auflistung von pulmonalen und extrapulmonalen Erkrankungen, die chronischen Husten auslösen können. Die einzelnen Krankheiten sind gemäß ihrer Inzidenz in drei Gruppen aufgeteilt.
Obstruktive Lungenerkrankung“) $[45,46]$, Husten als AsthmaÄquivalent (CVA - „Cough-variant Asthma“), eosinophiler Bronchitis [47] und von GÖR (gastro-ösophagealer Reflux) [48-53] sein. Chronischem Husten können auch seltene Krankheiten, wie die Sjögren-Krankheit, Granulomatose mit Polyangiitis (Wegenersche Granulomatose) [54], Colitis ulcerosa, Morbus Crohn [55 - 58], Morbus Horton (Arteriitis cranialis) [59] und Sarkoidose [60] zugrunde liegen.

Alters- und Geschlechtsspezifität von chronischem Husten Kinder leiden häufig an chronischem Husten, der jedoch oft mit einer guten Prognose einhergeht. Ursachen für chronischen Husten bei Kindern sind vielfältig. Es handelt sich hierbei um in- fektiöse und postinfektiöse bakterielle und virale Infekte der oberen und unteren Atemwege. Häufig ist es eine virale Bronchitis oder auch eine Infektion mit Bordetella pertussis, was Keuchhusten auslöst. Nichtinfektiöse Ursache des Hustens bei Kindern sind die häufig vorkommende chronisch-entzündliche Atemwegerkrankung Asthma bronchiale und der Gastro-Ösophageale Reflux. Seltene, aber ernstere Gründe für Husten treten bei Kindern mit kongenitalen Abnormalitäten des tracheobronchialen Systems, Immundefekt und Mukoviszidose auf. Psychische Faktoren können chronischen Husten verstärken oder diesen initiieren [61]. Über geschlechtsspezifische Unterschiede in der Prävalenz des chronischen Hustens wurde in einigen epidemiologischen Studien berichtet. Vom 2. bis zum 16. Lebensjahr leiden mehr 
Jungen als Mädchen an chronischem Husten. Dieses Ergebnis spiegelt die asthmatische Erkrankung der Kinder wider, die sich auch in Form von chronischem Husten manifestiert [62].

Hinsichtlich der geschlechtsspezifischen Unterschiede des chronischen Hustens ist bekannt, dass er vermehrt bei Frauen im mittleren Alter und zu Beginn der Menopause auftritt. Es handelt sich hierbei um einen idiopathischen Husten, bei dem nach umfangreichen diagnostischen Untersuchungen andere Ursachen ausgeschlossen werden konnten. Eine der möglichen Erklärungen für den chronischen Husten bei diesen Frauen ist die hormonelle Veränderung und die damit verbundene subklinische Inflammation der Atemwege [63,64].

Eine Studie berichtete, dass chronischer Husten bei Erwachsenen zwischen dem 18. -24. Lebensjahren mit Zigarettenkonsum vergesellschaftet ist [65]. Weiterhin kann chronischer Husten im mittleren Lebensalter (40.-49.Lebensjahr) Symptom gastrointestinaler Erkrankungen sein [66].

Aus der klinischen Erfahrung ist bekannt, dass der zirkadiane Rhythmus bei Patienten mit chronisch obstruktiven Lungenerkrankungen eine Rolle spielt. Patienten mit Asthma bronchiale klagen häufig über nächtlichen Husten und Hustenattacken in den frühen Morgenstunden. Dabei könnte eine erhöhte Aktivität des Parasympathikus für die bronchialen Obstruktionen verantwortlich sein. Leider gibt es dazu keine aussagekräftigen klinischen Daten.

\section{Medikamente als Husten-Initiatoren}

Medikamenten-Nebenwirkungen können zu Reizungen des Respirationstrakts führen $(\bullet$ Tab.3) und dadurch einen chronischen Husten herbeiführen. Wie genau Medikamente, wie u. a. Amiodaron, Ergotalkaloide, Methotrexat, Schleimlöser, inhalativ applizierte Corticosteroide und Zanamivir (weitere $\bullet$ Tab.3), Husten auslösen, ist bisher unbekannt [56, 67-69].

\begin{tabular}{|l|}
\hline ACE-Inhibitoren [98] \\
\hline Amiodaron [56, 68] \\
\hline Aspirin [58] \\
\hline$\beta$-Blocker [58] \\
\hline ß2-Sympathomimetika \\
\hline Zytostatika [69] \\
\hline Fentanyl [99] \\
\hline inhalierte Corticosteroide \\
\hline Interferon- $\alpha-2 a$ [100] \\
\hline Interferon- $\alpha$-2b [88] \\
\hline Ipratropiumbromid \\
Kontrazeptiva [67] \\
\hline Methotrexat [58] \\
\hline Mycophenolat [101] \\
\hline Nedocromil \\
\hline Nitrofurantoin [102] \\
\hline Nizatidin \\
\hline Pentamidin \\
\hline Propofol [103] \\
\hline Sirolimus [104] \\
\hline $\begin{array}{l}\text { oral oder systemisch } \\
\text { applizierte Mukolytika [105] }\end{array}$ \\
\hline Tiotropiumbromid \\
\hline Zanamivir \\
\hline
\end{tabular}

\section{ACE-Hemmer induziert Husten}

Die Inhibition des Angiotensin Converting Enzyms (ACE) führt einerseits zu einer Verminderung der Angiotensin-II-Produktion aus Angiotensin I und andererseits zu einer Hemmung von Abbau und Kumulation von Entzündungsmediatoren, wie Bradykinin und Prostaglandine. Angiotensin-II wirkt über Angiotensinrezeptoren $\left(A_{1} T_{1}\right.$ und $\left.A T_{2}\right)$ vasokonstriktorisch. Primär sinkt dadurch der periphere Blutgefäßtonus, und der Blutdruck nimmt ab. ACE-Hemmer werden daher zur Therapie von Bluthochdruck eingesetzt. Als Monotherapie und in Kombinationstherapie mit anderen Antihypertensiva sind ACE-Hemmer häufig Mittel der ersten Wahl. Als Nebenwirkung des durch ACE-Hemmer verminderten Abbaus sind erhöhte Konzentrationen von Bradykininen und Prostaglandinen, welche Husten über eine direkte Sensibilisierung der Hustenrezeptoren verursachen [10]. Dabei handelt es sich in erster Linie um einen trockenen Husten, der in den ersten drei Monaten bei 5-35\% der Patienten auftritt. Diese Nebenwirkung ist nicht dosisabhängig. Bei auftretendem Husten sollte der ACE-Hemmer abgesetzt bzw. gegen ein anderes Medikament entsprechend der Indikation ausgetauscht werden.

\section{Psychogener Husten}

Außerdem kann Husten auch durch psychische Störungen oder emotionale oder soziale Probleme ausgelöst werden [70-74]. Ein Beispiel hierfür ist die VCD (Vocal Cord Dysfunction), welche durch einen langen Krankheitsverlauf mit Unterbrechungen gekennzeichnet ist und neben psychischem auch physischen Stress zur Ursache haben kann. Typische Symptome der VCD, wie Atemnot, Husten und Pfeifen [75], entstehen durch inspiratorische Adduktion der Stimmbänder. VCD tritt häufig gemeinsam mit Asthma bronchiale auf.

Zuletzt kann Husten eine Reihe von Folgeerkrankungen verursachen. So können Rippenfrakturen durch Asthmaattacken, epileptische Anfälle, Hustensynkopen, Blutungen an den Konjunktiven, Epistaxis, GÖR, Petechien-Blutungen, mediadestinale Emphyseme, Kopf- und Brustschmerz und Harninkontinenz bei weiblichen Patienten entstehen [76].

Tab.3 Liste mögliche Medikamente und Wirkstoffe, die Husten auslösen können, in alphabetischer Reihenfolge (modifiziert [1]).

\section{Diagnostik von chronischem Husten}

Husten ist häufig ein Symptom von zahlreichen pulmonalen und auch extrapulmonalen Erkrankungen. Die Diagnostik von chronischem Husten stellt daher auch den erfahrenen Kliniker vor eine Herausforderung. Sie gestaltet sich als schwierig, wenn Husten als einziges Symptom auftritt und die Lungenfunktion und Bildgebung unauffällig sind. In diesem Zusammenhang kann Husten als häufiges Symptom bei isolierten Erkrankungen des tracheobronchialen Systems, bei Bronchiektasien und interstitiellen Lungenerkrankungen vorkommen. Bei interstitiellen Lungenerkrankungen ist bekannt, dass Husten auch als einziges Symptom der röntgenologisch pulmonalen Veränderungen um Monate vorausgehen kann. Eine detaillierte Anamnese und körperliche Untersuchung kann zu einer richtigen Diagnose verhelfen. Bei extrapulmonalen Erkrankungen sind organ- und fachspezifische Untersuchungen zur weiteren Differenzialdiagnostik erforderlich. 


\section{Therapie von Husten}

$\nabla$

Die Therapie von Husten kann - je nach Fall- und Krankheitsspezifität - mithilfe von Suppressiva, Expektorantien und Antiinflammatorika durchgeführt werden. Antiinflammatorische Medikamente, wie inhalativ und systemisch applizierte Corticosteroide und peroral verabreichte Leukotrien-Antagonisten, können Husten bei Asthma bronchiale und bronchialer Hyperreaktivität inhibieren [77]. Bei unproduktivem und trockenem Husten können zentral wirkende Substanzen Linderung verschaffen. Diese sind Morphium oder Codein bzw. deren Derivate wie Dextromethorphan und Pentoxyverin. Expektorantien wirken schleimlösend und bewirken eine Reizlinderung, allerdings werden ihre Effektivität und Wirksamkeit gegenwärtig kontrovers diskutiert. Zuckerkonzentratlösung (Zuckersirup, Honig) wirkt antitussiv, indem er sich auf den Hustenrezeptoren ablagert, diese bedeckt und für eine gewisse Zeitspanne verbleibt.

\section{Ausblick auf neue potenzielle Antitussiva \\ $\nabla$}

Die Forschung nach neuen Wirkstoffen gegen Husten hat in letzter Zeit einige mögliche Kandidaten hervorgebracht.

Je nach Zielmolekül gibt es bereits potenzielle Antitussiva. TRPA ${ }_{1}^{-}$ Rezeptoren können durch den TRPA ${ }_{1}$-Antagonist HC-030031 inhibiert werden (Wirkung nur im Tiermodell untersucht $[3,78$,

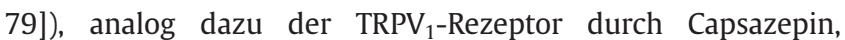
V112220 [80] und Iodresiniferatoxin (Effektiv im Tiermodell, Klinische Phasen I und II [81 -93]).

Bei einigen Substanzen wurde eine effektive Wirkung gegen Husten im Tiermodell gezeigt, allerdings existieren bisher keine Daten im Menschen. Zu diesen Substanzen gehören Nociceptin, welches den NOP1-Rezeptor aktiviert, die $\delta$ - und $\mu$-Opioidrezeptor-Agonisten, die an den peripheren Opioidrezeptoren wirken [94], am Tachykinin-Rezeptor wirkende NK-1-, NK-2- und NK-3-Antagonisten, der CB2-Agonist, welcher den Zitronensäure-induzierten Husten inhibiert, und die am Bradykinin- $B_{2}-R e-$ zeptor wirkenden $\mathrm{B}_{2}$-Rezeptor-Antagonisten, Icatibant und HOE-140 (inhibiert Husten, der durch ACE-Inhibitoren verursacht wird) [83].

Der GABA-Agonist Baclofen ist neben den $\mathrm{TRPV}_{1}$-Antagonisten das einzige bisher im Menschen getestete Medikament und zeigt gute Wirkung in einer Studie [95].

\section{Danksagung}

Diese Arbeit wird durch die DFG im Rahmen des Projektes DFG DI 1429 und die Stiftung „Bergmannhilfswerk Luisenthal“ an Prof. Dr. Quoc Thai Dinh und des Graduiertenkollegs 1441: Regulation der allergischen Entzündung in Lunge und Haut an Prof. Dr. Tobias Welte und Prof. Dr. Quoc Thai Dinh gefördert.

\section{Interessenkonflikt \\ $\nabla$}

Die Autoren geben an, dass kein Interessenkonflikt besteht.
Institute

${ }^{1}$ Klinik für Innere Medizin V - Pneumologie, Allergologie, Beatmungs- und Umweltmedizin, Universitätsklinikum und Medizinische Fakultät, Universität des Saarlandes, Homburg

2 Experimentelle Pneumologie und Allergologie, Universitätsklinikum und Medizinische Fakultät, Universität des Saarlandes, Homburg, Deutschland ${ }^{3}$ Zentrum für Innere Medizin, Klinik für Pneumologie, Medizinische Hochschule Hannover

\section{Literatur}

1 Kardos P, Berck H, Fuchs KH et al. [Guidelines of the german respiratory society for diagnosis and treatment of adults suffering from acute or chronic cough]. Pneumologie 2010; 64: 336-373

2 Widdicombe JG. Receptors in the trachea and bronchi of the cat. J Physiol 1954; 123: $71-104$

3 Taylor-Clark TE, Nassenstein C, McAlexander MA et al. TRPA1: a potential target for anti-tussive therapy. Pulm Pharmacol Ther 2009; 22: $71-74$

4 Canning BJ. Afferent nerves regulating the cough reflex: mechanisms and mediators of cough in disease. Otolaryngol Clin North Am 2010; 43: $15-25$, vii

5 Dinh QT, Groneberg DA, Peiser C et al. Expression of substance P and nitric oxide synthase in vagal sensory neurons innervating the mouse airways. Regul Pept 2005; 126: 189-94

6 Bolser DC, DeGennaro FC, O'Reilly S et al. Central antitussive activity of the NK1 and NK2 tachykinin receptor antagonists, CP-99,994 and SR 48968, in the guinea-pig and cat. Br J Pharmacol 1997; 121: 165-170

7 Bonham AC, Sekizawa S, Chen CY et al. Plasticity of brainstem mechanisms of cough. Respir Physiol Neurobiol 2006; 152: 312 - 319

8 Bonham AC, Sekizawa SI, Joad JP. Plasticity of central mechanisms for cough. Pulm Pharmacol Ther 2004; 17: 453 - 457

9 Braman SS, Corrao WM. Cough: differential diagnosis and treatment. Clin Chest Med 1987; 8: 177-188

10 Chung KF, Pavord ID. Prevalence, pathogenesis, and causes of chronic cough. Lancet 2008; 371: 1364-1374

11 Curley FJ, Irwin RS, Pratter MR et al. Cough and the common cold. Am Rev Respir Dis 1988; 138: 305 - 311

12 Footitt J, Johnston SL. Cough and viruses in airways disease: mechanisms. Pulm Pharmacol Ther 2009; 22: 108-113

13 McGarvey LP, Nishino T. Acute and chronic cough. Pulm Pharmacol Ther 2004; 17: 351 - 354

14 Birkebaek NH. Bordetella pertussis in the aetiology of chronic cough in adults. Diagnostic methods and clinic. Dan Med Bull 2001; 48: $77-80$

15 Widdicombe J. Functional morphology and physiology of pulmonary rapidly adapting receptors (RARs). Anat Rec A Discov Mol Cell Evol Biol 2003; 270: 2-10

16 Undem BJ, Carr MJ. Targeting primary afferent nerves for novel antitussive therapy. Chest 2010; 137: 177-184

17 Dinh QT, Mingomataj E, Quarcoo D et al. Allergic airway inflammation induces tachykinin peptides expression in vagal sensory neurons innervating mouse airways. Clin Exp Allergy 2005; 35: 820 - 825

18 Dinh QT, Suhling H, Fischer A et al. [Innervation of the airways in asthma bronchiale and chronic obstructive pulmonary disease (COPD)]. Pneumologie 2011; 65: 283-292

19 Doherty MJ, Mister R, Pearson MG et al. Capsaicin responsiveness and cough in asthma and chronic obstructive pulmonary disease. Thorax 2000; 55: $643-649$

20 Canning BJ. Central regulation of the cough reflex: therapeutic implications. Pulm Pharmacol Ther 2009; 22: 75 - 81

21 Eccles $R$. Central mechanisms IV: conscious control of cough and the placebo effect. Handb Exp Pharmacol 2009; 187: 241 - 262

22 Eccles $R$. The powerful placebo in cough studies? Pulm Pharmacol Ther 2002; 15: $303-308$

23 Emonds-Alt X, Proietto V, Steinberg $R$ et al. SSR240600 [(R)-2-(1-[2-[4[2-[3,5-bis(trifluoromethyl)phenyl]acetyl]-2-(3,4-dichlorophenyl) -2-morpholinyl]ethyl]- 4-piperidinyl)-2-methylpropanamide], a centrally active nonpeptide antagonist of the tachykinin neurokinin1 receptor: I. biochemical and pharmacological characterization. J Pharmacol Exp Ther 2002; 303: $1171-1179$

24 Chung KF. Cough: potential pharmacological developments. Expert Opin Investig Drugs 2002; 11: 955 -963

25 Lee KK, Birring SS. Cough and sleep. Lung 2010; 188 (Suppl. 01): S91 $\mathrm{S} 94$ 
26 Grace MS, Dubuis E, Birrell MA et al. Pre-clinical studies in cough research: Role of Transient Receptor Potential (TRP) channels. Pulm Pharmacol Ther 2013 http://dx.doi.org/10.1016/j.pupt.2013.02.007 (in press)

27 Raemdonck K, de Alba J, Birrell MA et al. A role for sensory nerves in the late asthmatic response. Thorax 2012; 67: 19-25

28 Preti D, Szallasi A, Patacchini R. TRP channels as therapeutic targets in airway disorders: a patent review. Expert Opin Ther Pat 2012; 22: $663-695$

29 O'Connell F, Thomas VE, Studham JM et al. Capsaicin cough sensitivity increases during upper respiratory infection. Respir Med 1996; 90: $279-286$

30 O'Hara J, Jones NS. "Post-nasal drip syndrome": most patients with purulent nasal secretions do not complain of chronic cough. Rhinology 2006; 44: 270 - 273

31 Lee PCL, Jawad MS, Eccles R. Antitussive efficacy of dextromethorphan in cough associated with acute upper respiratory tract infection. J Pharm Pharmacol 2000; 52: 1137 - 1142

32 Byard RW. Diphtheria - 'The strangling angel' of children. J Forensic Leg Med 2013; 20: 65 - 68

33 Groneberg-Kloft B, Dinh QT, Scutaru C et al. Cough as a symptom and a disease entity: scientometric analysis and density-equalizing calculations. J Investig Allergol Clin Immunol 2009; 19: 266-275

34 Dinh QT, Groneberg DA, Mingomataj E et al. Expression of substance P and vanilloid receptor (VR1) in trigeminal sensory neurons projecting to the mouse nasal mucosa. Neuropeptides 2003; 37: 245 250

35 Lee MG, Kollarik M, Chuaychoo B et al. Ionotropic and metabotropic receptor mediated airway sensory nerve activation. Pulm Pharmacol Ther 2004; 17: 355 - 360

36 Plevkova J, Brozmanova M, Pecova $R$ et al. Effects of intranasal capsaicin challenge on cough reflex in healthy human volunteers. J Physiol Pharmacol 2004; 55 : (Suppl. 03): 101 -106

37 Plevkova J, Varechova S, Brozmanova $M$ et al. Testing of cough reflex sensitivity in children suffering from allergic rhinitis and common cold. J Physiol Pharmacol 2006; 57: (Suppl. 04): 289-296

38 Pecova $R$, Vrlik $M$, Tatar $M$. Cough sensitivity in allergic rhinitis. J Physiol Pharmacol 2005; 56 (Suppl. 04): 171-178

39 Pratter MR. Chronic upper airway cough syndrome secondary to rhinosinus diseases (previously referred to as postnasal drip syndrome): ACCP evidence-based clinical practice guidelines. Chest 2006; 129: $63 \mathrm{~S}-71 \mathrm{~S}$

40 Lee $P$, Eccles $R$. Cough induced by mechanical stimulation of the upper airway in humans. Acta Otolaryngol 2004; 124: 720-725

41 Sanu A, Eccles R. Postnasal drip syndrome. Two hundred years of controversy between UK and USA. Rhinology 2008; 46: 86-91

42 Macedo $P$, Saleh $H$, Torrego A et al. Postnasal drip and chronic cough: An open interventional study. Respir Med 2009; 103: 1700-1705

43 Phelan PD. Postnasal drip and chronic cough. Lancet 1978; 2: 1309

44 Fujimura M, Sakamoto S, Kamio Yet al. Cough receptor sensitivity and bronchial responsiveness in normal and asthmatic subjects. Eur Respir J 1992; 5: 291 - 295

45 Smith J, Woodcock A. Cough and its importance in COPD. Int J Chron Obstruct Pulmon Dis 2006; 1: $305-314$

46 Terada K, Muro S, Ohara T et al. Cough-reflex sensitivity to inhaled capsaicin in COPD associated with increased exacerbation frequency. Respirology 2009; 14: 1151 - 1155

47 Fujimura $M$, Ogawa $H$, Nishizawa Y et al. Comparison of atopic cough with cough variant asthma: is atopic cough a precursor of asthma? Thorax 2003; 58: $14-18$

48 Birring SS, Pavord ID. Chronic cough and gastro-oesophageal reflux. Thorax 2004; 59: 633-634

49 Irwin RS, Zawacki JK, Curley FJ et al. Chronic cough as the sole presenting manifestation of gastroesophageal reflux. Am Rev Respir Dis 1989; 140: $1294-1300$

50 Kastelik JA, Jackson W, Davies TW et al. Measurement of gastric emptying in gastroesophageal reflux-related chronic cough. Chest 2002; 122: $2038-2041$

51 Irwin RS, French CL, Curley FJ et al. Chronic cough due to gastroesophageal reflux. Clinical, diagnostic, and pathogenetic aspects. 1993. Chest 2009; 136: e30

52 Morice AH. Gastro-oesophageal reflux and tachykinins in asthma and chronic cough. Thorax 2007; 62: $468-469$
53 Galmiche JP, Zerbib F, Bruley de Varannes S. Review article: respiratory manifestations of gastro-oesophageal reflux disease. Aliment Pharmacol Ther 2008; 27: 449-464

54 Zubairi $A B$, Liaquat HB, Husain SJ et al. Wegener's granulomatosis: a diagnostic challenge. J Pak Med Assoc 2009; 59: 853 - 855

55 Brown KK. Chronic cough due to chronic interstitial pulmonary diseases: ACCP evidence-based clinical practice guidelines. Chest 2006; 129: $180 \mathrm{~S}-185 \mathrm{~S}$

56 Daba $M H$, El-Tahir KE, Al-Arifi MN et al. Drug-induced pulmonary fibrosis. Saudi Med J 2004; 25: 700 - 706

57 Patel KN, Patel F, Hudgins L. Lofgren's syndrome presenting as a case of fever of unknown origin. Tenn Med 2007; 100: 37 - 38

58 Javidan-Nejad C, Bhalla S. Bronchiectasis. Radiol Clin North Am 2009; 47: $289-306$

59 Akers SM, Bartter TC, Pratter MR. Chronic cough as the sole manifestation of Hodgkin's disease. Chest 1992; 101: 853-854

60 Lynch JPIII, Kazerooni EA, Gay SE. Pulmonary sarcoidosis. Clin Chest Med 1997; 18: 755 - 785

61 de Jongste JC, Shields MD. Cough. 2: Chronic cough in children. Thorax 2003; 58: 998 - 1003

62 Wright AL, Stern DA, Kauffmann F et al. Factors influencing gender differences in the diagnosis and treatment of asthma in childhood: the Tucson Children's Respiratory Study. Pediatr Pulmonol 2006; 41: $318-325$

63 Mund E, Christensson B, Gronneberg R et al. Noneosinophilic CD4 lymphocytic airway inflammation in menopausal women with chronic dry cough. Chest 2005; 127: 1714-1721

64 Mund E, Christensson B, Larsson K et al. Sex dependent differences in physiological ageing in the immune system of lower airways in healthy non-smoking volunteers: study of lymphocyte subsets in bronchoalveolar lavage fluid and blood. Thorax 2001; 56: $450-455$

65 An LC, Berg CJ, Klatt CM et al. Symptoms of cough and shortness of breath among occasional young adult smokers. Nicotine Tob Res 2009; 11: 126-133

66 Ford AC, Forman D, Moayyedi P et al. Cough in the community: a cross sectional survey and the relationship to gastrointestinal symptoms Thorax 2006; 61: 975 - 979

67 Ben-Noun L. Drug-induced respiratory disorders: incidence, prevention and management. Drug Saf 2000; 23: 143 - 164

68 Rosenow ECIII. Drug-induced pulmonary disease. J Pract Nurs 1979; 29: $23-26,43$

69 Costabel $U$. [Drug-induced lung changes in rheumatology]. Z Rheumatol 1990; 49: 284-290

70 Ludviksdottir D, Bjornsson E, Janson C et al. Habitual coughing and its associations with asthma, anxiety, and gastroesophageal reflux. Chest 1996; 109: $1262-1268$

71 Erenberg G. Psychogenic cough. Pediatrics 2001; 108: 819-820

72 Niggemann $B$. How to diagnose psychogenic and functional breathing disorders in children and adolescents. Pediatr Allergy Immunol 2010; 21: $895-859$

73 Ishizaki Y, Kobayashi Y, Kino M. Chronic and persistent cough related to vulnerability to psychological stress: tic or psychogenic? Pediatr Int 2008; 50: $392-394$

74 Ramanuja S, Kelkar P. Habit cough. Ann Allergy Asthma Immunol 2009; 102: 91 - 95

75 Christopher KL, Wood RP, Eckert RC et al. Vocal-cord dysfunction presenting as asthma. N Engl J Med 1983; 308: 1566-1570

76 Morice AH. Epidemiology of cough. Pulm Pharmacol Ther 2002; 15 : $253-259$

77 Laitinen LA, Laitinen A, Haahtela T. A comparative study of the effects of an inhaled corticosteroid, budesonide, and a beta 2-agonist, terbutaline, on airway inflammation in newly diagnosed asthma: a randomized, double-blind, parallel-group controlled trial. J Allergy Clin Immunol 1992; 90: 32-42

78 Andre E, Gatti R, Trevisani $M$ et al. Transient receptor potential ankyrin receptor 1 is a novel target for pro-tussive agents. Br J Pharmacol 2009; 158: 1621 - 1628

79 Grace MS, Belvisi MG. TRPA1 receptors in cough. Pulm Pharmacol Ther $2011 ; 24: 286-288$

80 Leung SY, Niimi A, Williams AS et al. Inhibition of citric acid- and capsaicin-induced cough by novel TRPV-1 antagonist, V112220, in guinea-pig. Cough 2007; 3: 10

81 McLeod RL, Correll CC, Jia Y et al. TRPV1 antagonists as potential antitussive agents. Lung 2008; 186 : (Suppl. 01): S59-S65 
82 Szallasi A, Cortright DN, Blum CA et al. The vanilloid receptor TRPV1: 10 years from channel cloning to antagonist proof-of-concept. Nat Rev Drug Discov 2007; 6: 357-372

83 Gatti R, Andre E, Barbara C et al. Ethanol potentiates the TRPV1-mediated cough in the guinea pig. Pulm Pharmacol Ther 2009; 22: $33-$ 36

84 Groneberg DA, Niimi A, Dinh QT et al. Increased expression of transient receptor potential vanilloid-1 in airway nerves of chronic cough. Am J Respir Crit Care Med 2004; 170: 1276-1280

85 Gunthorpe MJ, Chizh BA. Clinical development of TRPV1 antagonists: targeting a pivotal point in the pain pathway. Drug Discov Today 2009; 14: 56-67

86 Gunthorpe MJ, Szallasi A. Peripheral TRPV1 receptors as targets for drug development: new molecules and mechanisms. Curr Pharm Des 2008; 14: $32-41$

87 Hillis BR. The assessment of cough suppressing drugs. Lancet 1952; 1 : $1230-1235$

88 Jia Y, McLeod RL, Wang X et al. Anandamide induces cough in conscious guinea-pigs through VR1 receptors. Br J Pharmacol 2002; 137: $831-836$

89 Materazzi S, Nassini R, Gatti R et al. Cough sensors. II. Transient receptor potential membrane receptors on cough sensors. Handb Exp Pharmacol 2009: 49-61

90 Jia Y, McLeod RL, Hey JA. TRPV1 receptor: a target for the treatment of pain, cough, airway disease and urinary incontinence. Drug News Perspect 2005; 18: $165-171$

91 Morice AH, Geppetti P. Cough. 5: The type 1 vanilloid receptor: a sensory receptor for cough. Thorax 2004; 59: 257-258

92 Trevisani M, Milan A, Gatti R et al. Antitussive activity of iodo-resiniferatoxin in guinea pigs. Thorax 2004; 59: 769-772

93 Caterina MJ, Julius $D$. The vanilloid receptor: a molecular gateway to the pain pathway. Annu Rev Neurosci 2001; 24: 487-517

\section{Hinweis:}

Dieser Artikel wurde geändert gemäß Erratum vom 27.6.2013.
94 Pavord ID, Chung KF. Management of chronic cough. Lancet 2008; 371: $1375-1384$

95 Dicpinigaitis PV, Dobkin JB. Antitussive effect of the GABA-agonist baclofen. Chest 1997; 111: 996-999

96 Takemura M, Quarcoo D, Niimi A et al. Is TRPV1 a useful target in respiratory diseases? Pulm Pharmacol Ther 2008; 21: 833-839

97 Groneberg-Kloft B, Feleszko W, Dinh OT et al. Analysis and evaluation of environmental tobacco smoke exposure as a risk factor for chronic cough. Cough 2007; 3: 6

98 Moore $N$, Noblet $C$, Joannides $R$ et al. Cough and ACE inhibitors. Lancet 1993; 341: 61

99 Lin CS, Sun WZ, Chan WH et al. Intravenous lidocaine and ephedrine, but not propofol, suppress fentanyl-induced cough. Can J Anaesth 2004; 51: 654-659

100 Slavenburg S, Heijdra YF, Drenth JP. Pneumonitis as a consequence of (peg)interferon-ribavirin combination therapy for hepatitis C: a review of the literature. Dig Dis Sci 2010; 55: 579-585

101 Elli A, Aroldi A, Montagnino G et al. Mycophenolate mofetil and cough. Transplantation 1998; 66: 409

102 Boggess KA, Benedetti TJ, Raghu G. Nitrofurantoin-induced pulmonary toxicity during pregnancy: a report of a case and review of the literature. Obstet Gynecol Surv 1996; 51: 367-370

103 Mitra S, Sinha PK, Anand LK et al. Propofol-induced violent coughing. Anaesthesia 2000; 55: 707-708

104 Roberts RJ, Wells AC, Unitt $E$ et al. Sirolimus-induced pneumonitis following liver transplantation. Liver Transpl 2007; 13: 853-856

105 Mallet P, Mourdi N, Dubus JC et al. Respiratory paradoxical adverse drug reactions associated with acetylcysteine and carbocysteine systemic use in paediatric patients: a national survey. PLoS One 2011; 6: e22792 